\title{
Research of Imaging Interpretation Model of CAT Logging Data
}

\author{
Dong Yong ${ }^{1 *}$ and Li Mengxia ${ }^{2}$ \\ ${ }^{* 1}$ School of Information and Mathematics, Yangtze University, Jingzhou Hubei 434023, China \\ ${ }^{2}$ Petroleum Engineering College, Yangtze University, Wuhan Hubei 430100, China \\ ${ }^{3}$ The Branch of Key Laboratory of CNPC for Oil and Gas Production, Yangtze University, Wuhan \\ Hubei 430100, China
}

Email: dongyong80@126.com

\begin{abstract}
The capacitance array tool (CAT) uses twelve probes distributed symmetrically to measure the response at different azimuths in the wellbore. It is especially suitable to measure highly-deviated wells and horizontal wells, and can intuitively reflect the phase distribution of fluid in the wellbore to image the logging information. This paper considers the dynamic subdivision of instrument rotation, and an imaging algorithm based on the modified Gaussian weighting function is proposed. This algorithm uses different parameters, vertically and horizontally, to adapt to the stratification phenomenon of fluid. In order to improve adaptability, the algorithm introduces the correction coefficient which is determined by using optimization. The results show that the imaging effect coincides well with the corresponding photographs.
\end{abstract}

Keywords: Production logging, CAT, Flow imaging, Gaussian weighting function, Correction coefficient.

\section{INTRODUCTION}

In highly-deviated wells and horizontal wells, the traditional production logging instrument has a large error rate [1] because of the natural stratification of fluid caused by gravitational differentiation in the case of multiphase flow. For this reason, the companies Halliburton and SONDEX jointly launched the capacitance array tool, or CAT. The CAT has twelve mini probes distributed radially in the wellbore and located in the same lateral section. Each probe can exactly probe the hold-up or attribute value of fluid around it with a detection range of $0.3 \mathrm{~mm}$ [2]. When the flow pattern in the wellbore is laminar flow, the probe can exactly offer hold-up of each phase. The CAT can process continuous measurement and fixed-point measurement and visually display the measured data from each of the twelve probes showing the distribution state of fluid in the wellbore. But research of the CAT imaging algorithm is in the preliminary stage and lacks relevant achievements. It is therefore necessary to further study the flow imaging algorithm of the CAT logging data.

Because of the small detection range of the probe relative to the wellbore radius, the response values of the twelve probes can be considered to show the attribute values at twelve points in this cross section for one cross section of the wellbore. Imaging is essentially interpolation.

It is difficult to obtain interpolation results with clear practical significance based solely on the attribute values at twelve probes without adding other information. Supposing that the relevance between the points continuously decreases with the increasing of the distance and that the flow pattern in the wellbore is laminar flow, then the speed of the continuous decrease of relevance in the horizontal direction with the distance increasing can be considered to be less than the one in the vertical direction. That is, the degree of approximation between the attribute value of a probe and neighboring points in the horizontal direction is great, and that of the vertical direction is relatively small. The efficient numerical simulation technique can also be used to analyze the distribution characteristics of multiphase flow in wellbores [3-4]. Hence, the inverse distance weighting method or the gauss weighting interpolation can be modified slightly and applied to the flow imaging algorithm of the CAT logging data. This paper discusses the modified gauss weighting interpolation, and uses the developed program to process the measured data. Finally, the algorithm performance is verified.

\section{ROTATION OF CAT AND SUBDIVISION OF WELLBORE CROSS SECTION}

In actual measurement, it is difficult to prevent the instrument from rotating. For the CAT, a special piece of equipment records the rotation angle in a timely manner relative to the upper part of the wellbore to determine the exact location of the CAT in the wellbore. Considering its advantageous properties, the Delaunay triangular mesh generation is chosen to subdivide the wellbore cross section. 
A variety of methods are available to realize the subdivision. subdivides the wellbore cross section without considering the rotation of the instrument, and then matches the probe to the boundary points. This method, however, has two deficiencies. The first drawback is that the twelve probes equally distributed on the circumference usually cannot satisfy the requirement of equidistance as they are matched to the boundary points. The second drawback is that the actual position of the probes do not always coincide with the boundary points. Hence, it is not feasible to initially subdivide the wellbore cross section. Under the premise of considering the rotation of instrument, this paper dynamically subdivides the wellbore to overcome the deficiencies of the method in reference [5]. The actual idea is described as follows.

The radius is taken along the direction of the rotated No.1 probe. Along the radius direction, the cross-sectional region is equally divided into $N$ annulus. In this paper, $N$ is taken as 30 . For each annulus, it is equally divided into $12 t$ for $t=1,2,3, \cdots, N$ in counterclockwise direction from the inside out. In this way, the cross-sectional region has $6 N(N+1)+1$ nodes in total. The probes are precisely positioned in the subdivision nodes and are distributed symmetrically.

\section{MODIFIED GAUSS INTERPOLATION ALGORITHM}

The imaging interpolation algorithm of the CAT is used to evaluate the measured values of other nodes on the cross section based on the values of nodes located in the twelve probes. The modified gauss weighting interpolation algorithm is described as Eq (1).

$w_{i}=\sum_{j=1}^{12} k_{j} \times D_{i j} \times T_{j}$

where $w_{i}$ is the response predicted value on the $i$-th node; $T_{j}$ is the measured response value of the $j$-th probe of CAT; $D_{i, j}$ is the contributive weight value of the $j$-th probe acting on the $i$-th node; and $k_{j}$ is the correction coefficient of the $j$ th probe. Because this paper adopts dynamic subdivision, the weight can only be determined dynamically because it depends on the subscripts $i$ and $j$. The determinative rules for weight $D_{i j}$ and correction coefficient $k_{j}$ are given below.

The Cartesian coordinates $O x y$ in the cross section plane are then established with the origin, denoted by $O$, as the core of the section, and the horizontal direction as the $x$-axis. The weight $D_{i j}$ is expressed as Eq (2).

$$
D_{i, j}=\exp \left(-\left(\frac{x-a}{m}\right)^{2}-\left(\frac{y-b}{n}\right)^{2}\right)
$$

where $m$ and $n$ are the diminishing control coefficients in the horizontal and vertical directions respectively; $a$ and $b$ are firstly, the method used in reference [5] is analyzed, which respectively the $x$-coordinate and $y$-coordinate of the node corresponding to the $j$-th probe; $x$ and $y$ are the $x$ coordinate and $y$-coordinate of the $i$-th node.

The interpretation model is expected to be effective for every node with certain applicability. So it is essential that the computational results at the nodes are close to the measured values. But it is not sufficient to only use the above rule to determine $D_{i, j}$. For this reason, the correction coefficient $k_{j}$ is introduced to modify the weight of the probe to guarantee that the computational result at the probe position equals the measured value or that the error between the two is very small.

In order to determine the correction coefficients $k_{1}, k_{2}, \cdots, k_{12}$, the objective function is established, shown as $\mathrm{Eq}(3)$.

$\min \sum_{j=1}^{12}\left(w_{j}-T_{j}\right)^{2}$

where $w_{j}$ is the computational value at the node of $j$-th probe obtained from Eq (1). The optimal algorithm [6-10] is needed to determine the correction coefficients. The flow chart of PSO is shown in Figure 1. When the objective function arrives at the minimum value, the corresponding correction coefficients can be determined.

For the horizontal well and highly-deviated well, the well inclination, flow rate and the inflow of fluid all have an influence on the flow regime in the wellbore. The $m$ and $n$ values are determined according to the pre-judging of the wellbore diameter and flow regimes to guarantee that the change rule of $D_{i, j}$ reflects the change rule of fluid distribution in the wellbore. The difference between the above two change rules is modified by choosing $k_{1}, k_{2}, \cdots, k_{12}$. Thus the modified Gaussian weighting algorithm can adapt to the flow regime in the wellbore and the well inclination.

\section{TRANSFORMATION FROM ATTRIBUTE VALUE OF NODE TO ATTRIBUTE VALUE OF COLOR}

The transformation from the response value of node to the attribute value of color must first be realized in order to visualize the cross section of the wellbore. The theoretical response values of CAT probe in water, oil and gas phase are $1,0.2$ and 0 respectively [2]. While dealing with the response values, a neighborhood of the theoretical response value can be assigned. As long as the response value locates in the corresponding neighborhood, we consider the corresponding phase. This is also the imaging method used for the point diagram in the third bars in Table. 1. RGB color format is used [11]. Blue $(0,0,255)$ is used to show the water phase, green $(0,255,0)$ to show the oil phase and red $(255,0,0)$ to show the gas phase. 


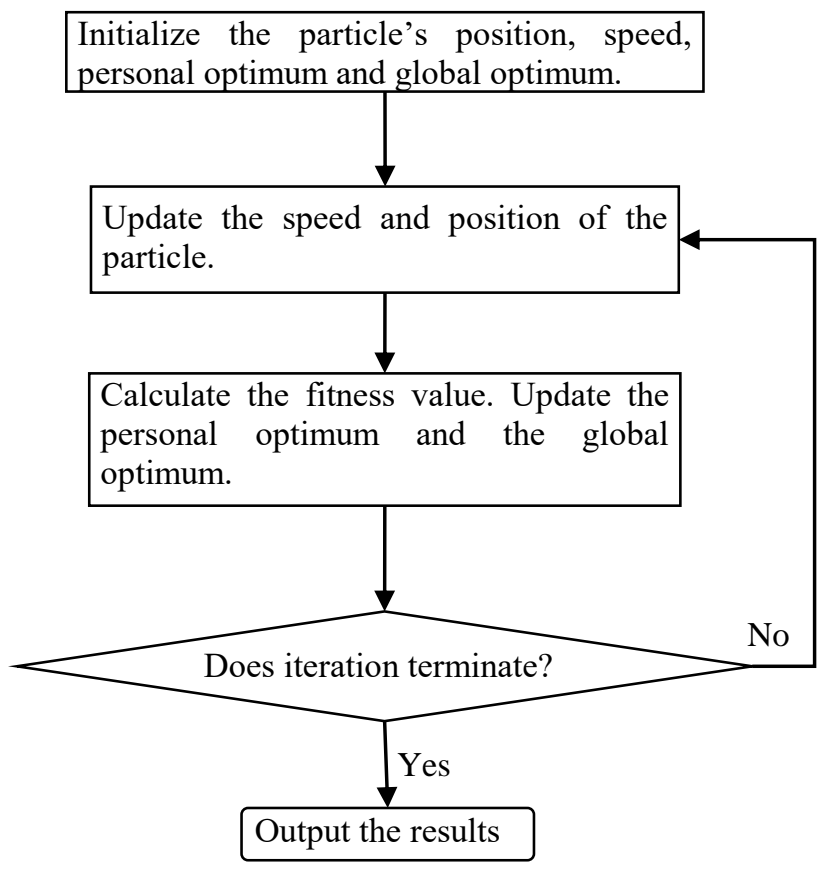

Figure 1. Flow chart of PSO

\section{APPLICATION}

The data comes from the gas, oil and water three-phase flow experiment based on the multiphase flow simulator. The pipe diameter is $120 \mathrm{~mm}$. The temperature in the experiment is $12-13^{\circ} \mathrm{C}$; pressure is $0.2 \mathrm{MPa}$; mediums are tap water, diesel and gas; well inclination is $90^{\circ}$; gas flow rate is 100 $\mathrm{m}^{3} / \mathrm{d}$; total flow rate of oil and water is $150 \mathrm{~m}^{3} / \mathrm{d}$; water content is $80 \%$. CAT instrument is dragged to measure at different rates in the pipeline.

According to the foregoing modified Gaussian weighting algorithm, taking $m=62$ and $n=24$, APSO is used to calculate the correction coefficient. The imaging proceeds under different measurement modes which include spot measurement denoted as 0 , velocity measurement $4 \mathrm{~m} / \mathrm{min}$ denoted as $4 \mathrm{U}$, velocity measurement $12 \mathrm{~m} / \mathrm{min}$ denoted as $12 \mathrm{U}$ and velocity measurement $20 \mathrm{~m} /$ min denoted as $20 \mathrm{U}$. The imaging results and the corresponding profile images are shown in Table 1, where the phase distribution of the cross section can be intuitively seen. Although the measurement mode has a certain influence on the measured results, the change is small. The distribution of multiphase fluid in the wellbore is largely consistent with the photograph.

\section{CONCLUSIONS}

(1) Twelve probes of CAT measure the holdup of phase along different directions on cross section of wellbore for the horizontal well and highly-deviated well. The imaging interpretation can intuitively display the phase distribution on the cross section.

(2) Through introducing the correction coefficient and adopting the special subdivision ways for the wellbore cross section, the interpretation model proposed in this paper improves the precision of the interpretation while considering the rotation of the instrument.

\section{REFERENCES}

(3) The consistency between the imaging results and photographs shows that the interpretation model proposed in this paper is valid.

(4) We builds imaging interpretation model for the wellbore cross section in this paper. But we don't consider the one for one segment of the wellbore. We can consider to use the lattice Boltzmann method [12-15] to build priori knowledge, and then construct three-dimensional imaging interpretation model.

Table 1. Comparison between imaging results of CAT experiment data and the corresponding photographs under different measurement modes

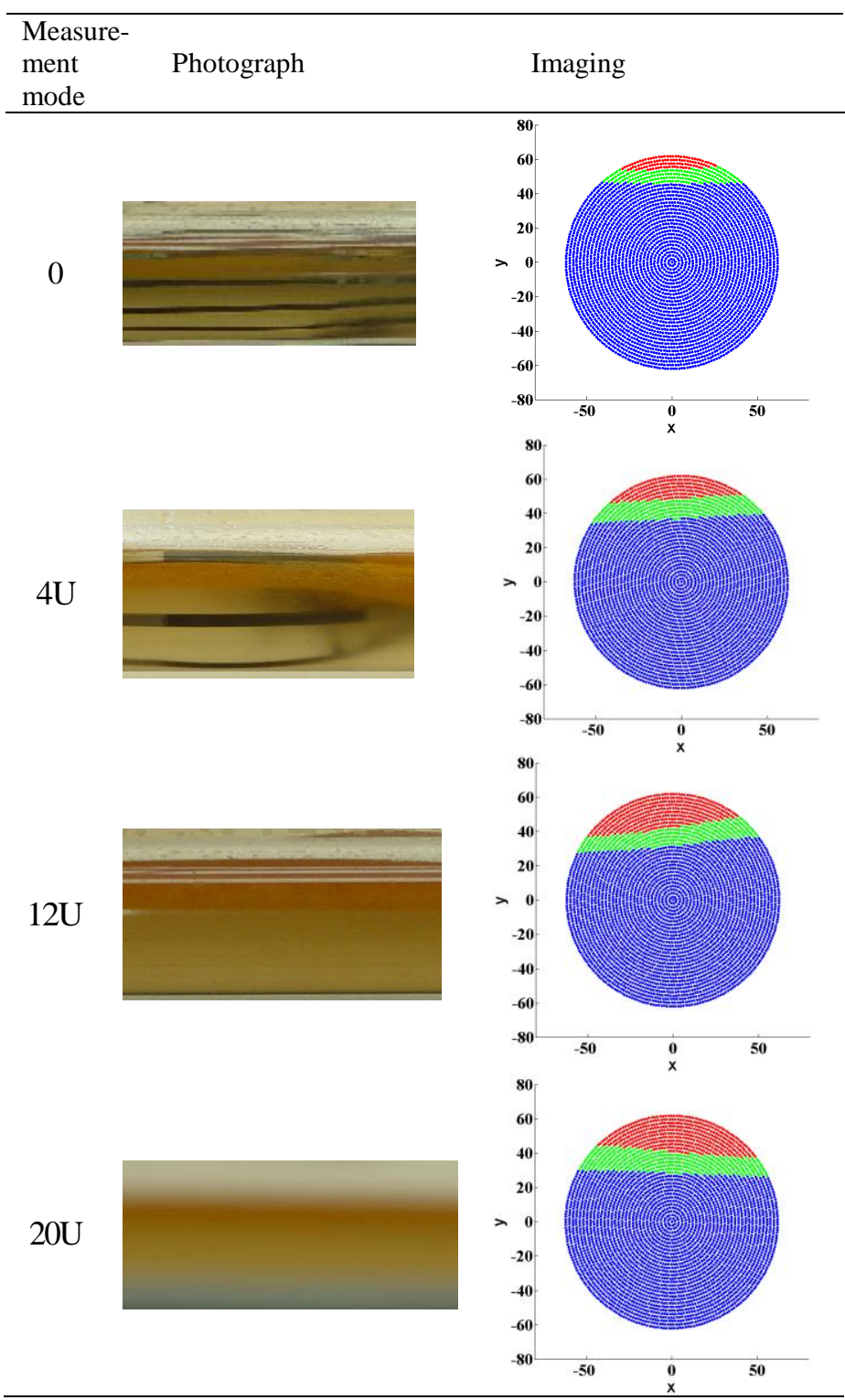

\section{ACKNOWLEDGMENT}

The authors wish to thank technicians in the Branch of Key Laboratory of CNPC for Oil and Gas Production for their great help. This paper is supported by the Educational Commission of Hubei Province of China (B2015449) and the National Natural Science Foundation of China (61572084), Hubei Provincial Natural Science Foundation of China (2013CFA053) and National Natural Science Foundation of China (61273179 and 61572084). 
[1] GUO Haimin, Introduction of Production Logging. Beijing, China: Petroleum Industry Press, 2003, pp. 522-532.

[2] Gary Frisch, Tegwyn Perkins h and John Quirein, "Integrating wellbore flow images with a conventional production log interpretation method," presented at the SPE ATCE, San Antonio, America, Sep. 29-Oct. 2, 2002.

[3] Zeghbid and R. Bessaïh, "Mixed convection in liddriven cavities filled with a nanofluid," Int J Heat \& Tech, vol. 33, no. 4, pp. 77-84, Dec. 2015. DOI: 10.18280/ijht.330410.

[4] Haixia Li, Bin Li and Xue Bai, "Three dimensional modeling of gas-solid coupled free and porous flow in a filtretion process," Int J Heat \& Tech, vol. 33, no. 4, pp. 101-106, Dec. 2015. DOI: 10.18280/ijht.330413.

[5] Dai Jacai, Guo Haimin, Liu Heng, Yang Zhengfa, Qin Minjun and Lu Lijun, "The flow imaging algorithm study on logging data of capacitor array tool," Well Logging Technology, vol. 34, no. 1, pp. 27-30, Mar. 2010. DOI: $10.3969 /$ j.issn.1004-1338.2010.01.006.

[6] Tao Lin, Peng Wu, Fengmei Gao, Yi Yu and Linhong Wang, "Study on SVM Temperature compensation of liquid ammonia volumetric flowmeter based on variable weight PSO," Int J Heat \& Tech, vol. 33, no 2, pp. 151-156, Jun. 2015. DOI: 10.18280/ijht.330224.

[7] DONG Yong and GUO Haimin, "Adaptive chaos particle swarm optimization baesd on colony fitness variance," Application Research of Computers, vol. 28, no. 3, pp. 854-856, Mar. 2011. DOI: 10.3969/j.issn.1001-3695.2011.03.015.

[8] E. Mehdizadeh and A. Fatehi Kivi, "Three metaheuristic algorithms for the single-item capacitated lotsizing problem," IJE Transactions B: Applications, vol. 27, no. 8, pp. 1223-1232, Aug. 2014. DOI: 10.5829/idosi.ije.2014.27.08b.08.

[9] U. Bahalke, A. M. Yolmeh, A. N. Hajizade and A. Bahalke. "Genetic and tabu search algorithms for the single machine scheduling problem with sequence dependent set-up times and deteriorating jobs," IJE Transactions A: Basis, vol. 23, no. 3\&4, pp. 227-234, Nov. 2010. DOI: 10.5829/idosi.ije.2010.23.34a.03.

[10] R. Tavakkoli-Moghaddam, M. Heydar and S. M. Mousavi, "A hybrid genetic algorithm for a biobjective scheduling problem in a flexible manufacturing cell," IJE Transactions A: Basis, vol. 23, no. 3\&4, pp. 235-252, Nov. 2010. DOI: 10.5829/idosi.ije.2010.23.34a.04.

[11] Dong Yong, Wu Chuansheng, Lv Qinghe, Li Hongkui and Guo Haimin, "Study of $\mathrm{CO}_{2}$ fluid density calculation model based on grayscale image," Int $J$ Heat \& Tech, vol. 33, no. 1, pp. 151-156, Mar. 2015. DOI: $10.18280 /$ ijht.330122.
[12] Ahmed Mahmoudi and Imen Mejri, "Isothermal carbonization of wood particle: Application of the Lattice Boltzmann Method", Int J Heat \& Tech, vol.33, no.2, pp. 129-134, Jun. 2015. DOI: 10.18280/ijht.330221.

[13] Shouguang Yao, Xinwang Jia, Anjie Hu and Rongjuan Li. "Analysis of Nanofluids phase transition in pipe using the Lattice Boltzmann Method", Int J Heat \& Tech, vol. 33, no. 2, pp. 103-108, Jun. 2015. DOI: 10.18280/ijht.330217.

[14] Ahmed Mahmoudi and Imen Mejri, "Analysis of conduction-radiation heat transfer with variable thermal conductivity and variable refractive index: Application of the Lattice Boltzmann Method", Int J Heat \& Tech, vol. 33, no. 1, pp. 1-8, Mar. 2015. DOI: 10.18280/ijht.330101.

[15] Shouguang Yao, Xinwang Jia, Tao Huang and Luobin Duan. "Numerical simulation of bubble motion in boiling nanofluids based on Lattice Boltzmann Method", Int J Heat \& Tech, vol. 33, no. 1, pp. 71-76, Mar. 2015. DOI: 10.18280/ijht.330110.

\section{NOMENCLATURE}

$N \quad$ Dimensionless number of subdivision annulus

$t \quad$ Dimensionless number

$w \quad$ Dimensionless predicted attribute value of node

$k \quad$ Dimensionless correction coefficient of probe

D Dimensionless contributive weight value of the probe acting on the node

$T \quad$ Dimensionless measured response value of the probe

Oxy Cartesian coordinates in the cross section plane

$a \quad x$-coordinate of the node corresponding to the probe, $\mathrm{mm}$

$b \quad y$-coordinate of the node corresponding to the probe, $\mathrm{mm}$

$x \quad x$-coordinate of the node corresponding to the node, $\mathrm{mm}$

$y \quad y$-coordinate of the node corresponding to the node, $\mathrm{mm}$

Dimensionless control coefficients in the horizontal direction

$n \quad$ Dimensionless control coefficients in the vertical direction

\section{Subscripts}

$i \quad$ Dimensionless number of node

$j \quad$ Dimensionless number of probe 